WellBeing International

WBI Studies Repository

3-2001

\title{
Size Matters: Impact of Item Size and Quantity on Array Choice by Chimpanzees (Pan troglodytes)
}

Sarah T. Boysen

The Ohio State University

Gary G. Berntson

The Ohio State University

Kimberly L. Mukobi

The Ohio State University

Follow this and additional works at: https://www.wellbeingintlstudiesrepository.org/acwp_asie

Part of the Animal Studies Commons, Comparative Psychology Commons, and the Other Animal

Sciences Commons

\section{Recommended Citation}

Boysen, S. T., Berntson, G. G., \& Mukobi, K. L. (2001). Size matters: impact of item size and quantity on array choice by chimpanzees (Pan troglodytes). Journal of Comparative Psychology, 115(1), 106.

This material is brought to you for free and open access by WellBeing International. It has been accepted for inclusion by an authorized administrator of the WBI Studies Repository. For more information, please contact wbisr-info@wellbeingintl.org.

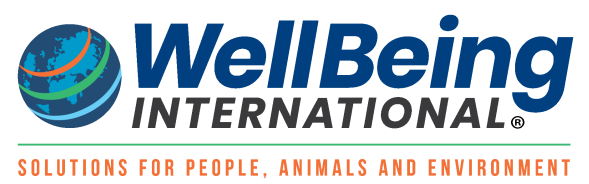




\title{
Size Matters: Impact of Item Size and Quantity on Array Choice by Chimpanzees (Pan troglodytes)
}

\author{
Sarah T. Boysen ${ }^{1,2}$, Gary G. Berntson ${ }^{1}$, and Kimberly L. Mukobi ${ }^{1}$ \\ ${ }^{1}$ Ohio State University \\ ${ }^{2}$ Emory University
}

\section{$\underline{\text { ABSTRACT }}$}

The authors previously reported that chimpanzees (Pan troglodytes) showed a striking bias to select the larger of 2 candy arrays, despite a reversed reward contingency in which the animals received the smaller, nonselected array as a reward, except when Arabic numerals were used as stimuli. A perceptual or incentive-based interference occurred that was overcome by symbolic stimuli. The authors of the present study examined the impact of element size in choice arrays, using 1 to 5 large and small candies. Five test-sophisticated chimpanzees selected an array from the 2 presented during each trial. Their responses were not optimal, as animals generally selected arrays with larger total mass; thus, they received the smaller remaining array as a reward. When choice stimuli differed in size and quantity, element size was more heavily weighted, although choices reflected total candy mass. These results replicate previous findings showing chimpanzees' difficulties with quantity judgments under reverse reward contingencies and also show that individual item size exerts a more powerful interference effect.

Previous studies of chimpanzees' ability to make relative size judgments of food items were first reported by Menzel $(1960,1961)$ and colleagues (Menzel \& Davenport, 1962; Menzel \& Draper, 1965). Results indicated that the chimpanzees could reliably select the largest portion from among the available choices without specific training. We have also found that relative quantities of stimuli are important determinants of choices by chimpanzees such that the disposition to select a larger quantity can interfere with the chimpanzees' selection of the smaller array when a reverse reward contingency is enforced. Under these conditions, to maximize reward, the chimpanzees should choose the smaller array, which would be discarded, and the chimpanzees would be rewarded with the remaining, larger array. This interference effect has been highly persistent over hundreds of trials, over several years, and through numerous versions of the task (see Boysen \& Berntson, 1995; Boysen, Berntson, Hannan, \& Cacioppo, 1996). If, however, arrays were replaced by number symbols (all chimpanzees had expertise with the functional use of Arabic numerals), the chimpanzees were able to reliably select the smaller numeral, and under the imposed reverse contingency, received the larger reward. This more optimal performance was shown immediately once numbers were used, although in subsequent sessions when candy arrays were reintroduced, all chimpanzees' performances reverted to the original strategy of choosing the larger array (Boysen et al., 1996). Results suggested that a powerful, perceptually driven predisposition was competing with the acquired associative effects of the reinforcement contingencies, and this interference 
effect has been replicated with three other species, including Japanese macaque monkeys (Silberburg \& Fujita, 1996), pigeons (Emmerton, 1995), and more recently, squirrel monkeys (Anderson, Awazu, \& Fujita, 2000).

The ability of older children and some nonhuman subjects to take into account the divergent perceptual features of a conservation task shares some of the same requirements of our quantity judgment task. However, in the quantity judgment task, there was no physical transformation of the stimuli during testing. Instead, the perceptual discrepancies were inherent in the physical and incentive properties of the comparison arrays, including quantity, valence, representational modality, or some combination of the three (Boysen \& Berntson, 1995; Boysen et al., 1996; Boysen, Berntson, \& Mukobi, 1999). Of further interest are other potential perceptual features such as size, which may also contribute to judgments between stimuli with inherent physical differences. In the present experiment, we examined the contribution of size to comparisons of food arrays, under the same reversed-contingency conditions as our original quantity judgment task.

\section{Method}

\section{Subjects}

Five adult chimpanzees (Pan troglodytes) served as subjects, including 2 females (ages 17.0 and 40.0 years), and 3 males (ages 11.0, 19.5, and 20.0 years). All chimpanzees had extensive experience and training on a variety of cognitive and behavioral tasks (e.g., Boysen et al., 1999; Kuhlmeier, Boysen, \& Mukobi, 1999), including counting with Arabic numerals (see Boysen, 1997). All chimpanzees had also participated in previous quantity judgment studies; however, all food items were a fixed size (e.g., Boysen \& Berntson, 1995; Boysen et al., 1996, 1999).

\section{Procedures}

All chimpanzees were tested individually in an indoor room connected to their outdoor play areas. The test room had a large, heavy polycarbonate window with an outside shelf where stimulus items were displayed. The chimpanzees made their choices by pointing to items, with the experimenter positioned out of view of the chimpanzee but able to clearly see their selections. Procedures were similar to previous studies (e.g., Boysen \& Berntson, 1995), during which the chimpanzees chose between two candy arrays and were subsequently reinforced with candy from the nonselected array. In the present study, arrays composed of large or small gumdrops (39 gm vs. $12 \mathrm{gm}$ ) were presented in two separate dishes on each trial. Individual arrays always consisted of a fixed element size (large or small candies), but the two separate dishes might contain an array composed of small candies in one or both dishes or large candies in one dish and smaller candies in the other. During each trial, the two dishes were placed on the shelf approximately $30 \mathrm{~cm}$ apart, and the chimpanzees were to select one array by pointing. After each choice, the experimenter removed the candy from the selected dish, and provided the chimpanzee with the candy from the other (nonchosen) dish. Thus, it was to the chimpanzee's advantage to choose the dish with the smaller number of candies to receive the larger remaining array (in the other dish) as a reward.

Chimpanzees were tested for 11 sessions, 16 trials per session, and all chimpanzees had the same number of trials and trial types, with all types presented equally often. All possible combinations of arrays composed of one to four small candies and one to three large candies were tested, two arrays per trial, with the exception that identical pairs, such as one small versus one small or three large versus three large, were not used. Additional arrays of up to five elements of both large and small candies were used, in later sessions. Order and left-right position of the arrays were counterbalanced across both trials and sessions. Trials in which a chimpanzee failed to make a choice or those in which the choice was in any 
way ambiguous were dropped from the analyses, although these represented less than $1 \%$ of all test trials.

\section{Data Analyses}

Because of the modest number of chimpanzees tested and because of the need to avoid distribution assumptions, primary analyses were based on nonparametric methods, including chi-square tests with the Yates correction for testing performance against chance, and chi-square tests for goodness-of-fit were used for all pairwise comparisons. In addition, a disparity ratio index was derived for each choice pair, as described in Boysen et al. (1996). This index was used because previous studies (e.g., Boysen et al., 1996; Kreps, 1988; Mischel, Shoda, \& Rodriguez, 1989; Schrier, 1956) had demonstrated that quantities and differences among quantities were judged relatively. The disparity ratio was derived from the absolute difference in magnitude (total array mass) between the two sets of choice stimuli, divided by the average total magnitude of both arrays, providing a Weber-like summary statistic that expressed the disparity between the two choices scaled by the overall magnitude. The relation between the disparity ratio and performance was further characterized by linear regression so that the slope of the disparity ratio-performance function could be defined (see Figure 1).

\section{$\underline{\text { Results }}$}

\section{Task Interference Effect: Replication and Extension With Fixed Element Sizes}

As observed previously, the chimpanzees had difficulty in maximizing payoff by selecting the smaller of two arrays of candies to obtain the larger remaining quantity. This was especially apparent on trials where the candy arrays both comprised equivalent element size (one to five items, either all small or all large candies). Optimal responses under these conditions were below chance, reflecting an inherent bias toward selection of the larger array; overall probability of an optimal response $=174 / 437$ trials $=40 \%$, $X^{2}(1, N=437)=18.1, p<.001$. This was true for choices between arrays comprising small candies, $81 / 220=37 \%, X^{2}(1, N=220)=15.3, p<.001$, and large candies, $93 / 217=43 \%, X^{2}(1, N=217)=18.1, p$ $<.001$, which did not differ from each other. This response bias was also observed for individual chimpanzees (percentage of correct responses: $29 \%$ to $43 \%$ ), with 1 exception, Bobby (the 11-year-old male); probability of optimal response $=56 / 88=64 \%, X^{2}(1, N=88)=6.6, p=.011$. Group performance (excluding Bobby) was more strongly biased, $118 / 349=34 \%, X^{2}(1, N=349)=36.6, p<.001$.

\section{Array Quantity and Disparity}

Task performance was not uniform across all choice pairs, but as was seen previously (see Boysen et al., 1996), was related to the disparity between the two arrays, as well as the overall size of the arrays. Again, the relative performance across array pairs was best described by the disparity ratio. Figure 1 illustrates task performance as a function of the disparity ratio index, which ranged from 0.22 for the four versus five candies to 1.33 for one versus five candies. As depicted by the overall group function (heavy line), performance became progressively worse with higher disparity ratios. Thus, the greater the relative reward differential, the more likely the chimps would select the larger array and then receive a smaller reward. Overall regression analysis revealed that the disparity ratio accounted for approximately $41 \%$ of variance in performance, slope $=-.14, R^{2}=.41, F(1,8)=5.45, p<.05$, and also accounted for more response variance than either the absolute array disparity $\left(R^{2}=.23\right)$ or the overall size of the arrays $\left(R^{2}=\right.$ .21). This ratio effectively captured the interacting effects of both variables, because the stepwise addition of size and disparity to the regression model did not significantly account for greater variance $\left(R^{2}=.44\right.$ vs $.41, n s)$. 
Figure 1. Performance depicted as a function of the disparity ratio (magnitude of the disparity between array pairs/average magnitude of the two arrays) of the comparison stimulus (candy) arrays.

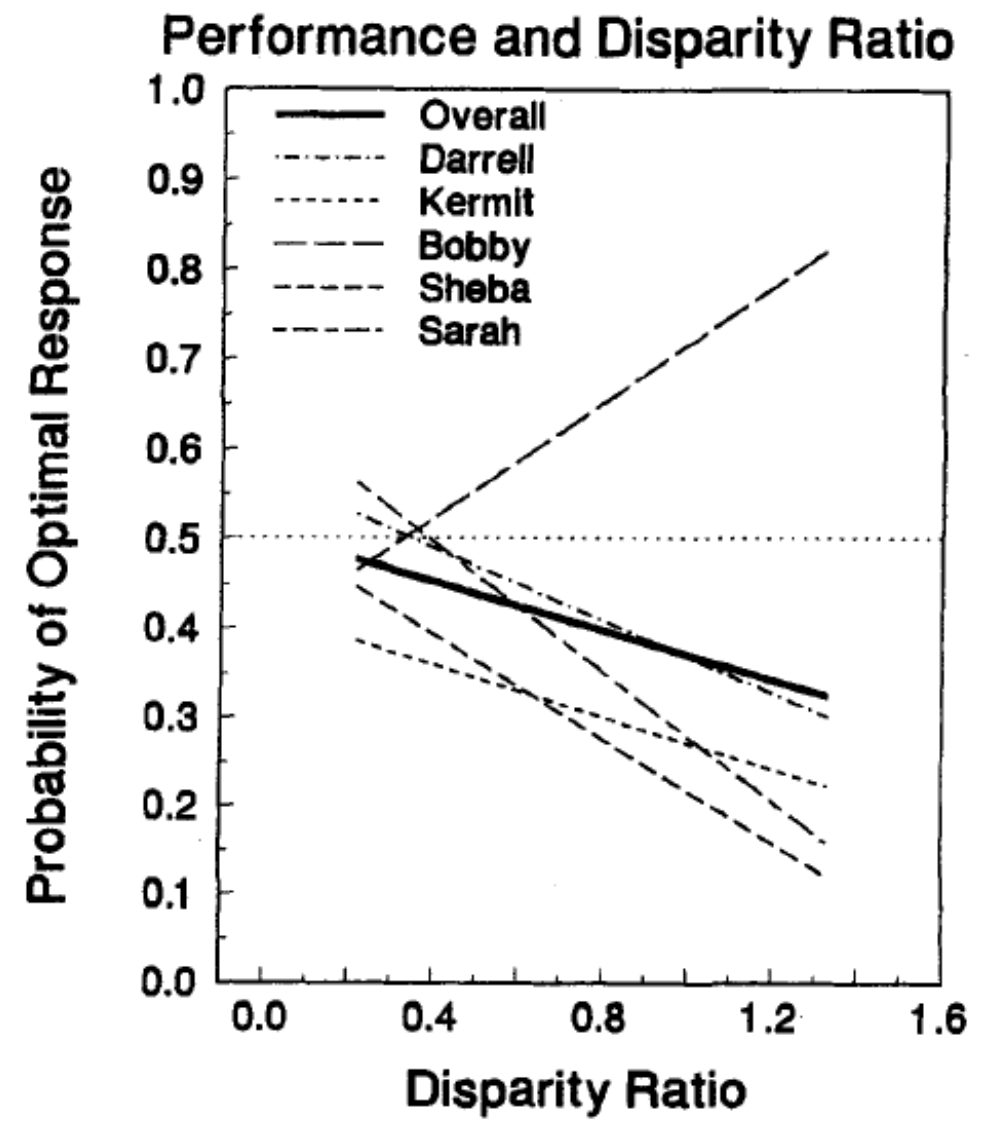

\section{Individual Differences and Interference Patterns}

As noted, 1 chimpanzee, Bobby, a young adult male, displayed performance that was significantly greater than chance compared with the other 4 chimpanzees. As shown in Figure 1, the slope of the disparity ratio-performance function was highly similar for these chimpanzees but was distinctly different for Bobby, whose responses resulted in a positive slope, $+0.32 ; R^{2}=.60, F(1,8)=12.20, p<.01$. Disparity ratioperformance slopes for the remaining 4 chimpanzees were all negative (-.15 to -.36). These differences indicated that Bobby was less affected by the response interference effect, and they showed improved performance with higher disparity ratios.

Categorical differences in individual chimpanzees' performances were further evaluated by chi-square tests for goodness-of-fit to test pairwise differences in performance among the chimpanzees. Results revealed that Bobby's overall performance was significantly better than any other chimpanzee, all $X^{2} s(1, N$ $>86)>7.4$, ps $<.01$, whereas the remaining chimpanzees did not differ from each other, all $X^{2} s(1, N>$ $86)<1.9$, ps $>$.17. Analyses of the disparity ratio-performance slopes further support two distinct performance categories. Pairwise comparisons among chimpanzees revealed that the positive slope for Bobby was significantly different from the negative slope of each of the other chimpanzees (all Fisher's zs $>2.79$, ps < .005), whereas none of the latter differed among themselves (all $z s<1, n s$ ). Because these findings suggested a categorical difference in Bobby's performance, separate analyses were run for Bobby and the remaining 4 chimpanzees. 
When analyzed separately, regression analyses revealed that the 4-chimpanzee group (Darrell, Kermit, Sheba, and Sarah) displayed an even more negative slope to the disparity ratio-performance function (-.26 vs. -.14 ), with this slope accounting for a greater proportion of variance, $58 \%$ versus $41 \%, R^{2}=.58$, $F(1,8)=10.98, p<.01$. As before, this contrasted sharply with the regression function for Bobby, slope $=$ $+.32, R^{2}=.60, F(1,8)=12.20, p<.01$.

\section{Size Matters}

Analyses and results of previous findings were based on choice pairs that had array elements of equal size and mass (see Boysen et al., 1996). In the present study, however, arrays were composed of elements of different mass. The overall results indicated that element size did affect choices, and this was apparent on trials where arrays were of equal number but one array was composed of large candies, and the other, small ones. With a condition of reversed reward contingency in effect, the chimpanzees could optimize payoff on these trials by selecting the array with smaller candy elements, thereby receiving the remaining array of larger candies. However, across all chimpanzees, performance on the trials was below chance, with the smaller size arrays selected, on only $42 \%$ of trials. In addition, distinct performance patterns emerged, with the performance of 1 chimp (Bobby) significantly better. Bobby was able to reliably select the array with smaller elements and thus garnered the larger reward mass on $95 \%$ of the trials, $19 / 20, X^{2}(1, N=20)=16.2, p<.001$. In contrast, performance of the remaining 4 chimpanzees was significantly below chance, with arrays with smaller elements selected on only 23 of 80 trials, $M=29 \%$, range $=25 \%-30 \%, X^{2}(1, N=80)=14.5, p<.001$. These findings indicated that element size did matter for all chimpanzees. Bobby, who had performed more optimally with the quantity judgment trials described above, was also able to better respond to arrays composed of differing element sizes. Thus, Bobby was able to reliably select the array composed of smaller candies and consequently received the larger remaining candy array for a reward. The 4 other chimpanzees displayed a tendency to select arrays that contained larger individual candies, and consequently these chimpanzees received the remaining array of smaller candies for their reward.

\section{Size Versus Quantity}

These findings suggest that both quantity and element size can contribute to quantity judgments and response choices by chimpanzees. This issue was addressed by evaluating total array magnitude and trial sets in which array pairs differed in element size as well as quantity. On such trials, a less than optimal response bias related to element size was apparent. Over all trials and chimpanzees, arrays containing larger candies were selected on $66 \%$ of trials, $225 / 339$ trials, $x^{2}(1, N=339)=36.3, p<.001$, and this bias was stable across sessions. ${ }^{1}$ With the exception of a single chimpanzee, the chimpanzees exhibited a tendency to select arrays containing larger elements, regardless of quantity or overall array magnitude. Excluding Bobby, arrays consisting of large candy elements were selected on $79 \%$ of the trials, range $=70 \%-90 \%$ overall, 214 of 271 trials, $X^{2}(1, N=271)=83.5, p<.001$. In contrast, Bobby again displayed the opposite pattern, selecting arrays containing larger elements on only $16 \%$ of the trials, $11 / 68$ trials, $X^{2}(1, N=68)=31.1, p<.001$.

Although array magnitude may have contributed to the bias, overall magnitude appeared to account for a small proportion of variance. Regression analyses revealed the expected negative slope of performance on magnitude ratio (magnitude disparity/mean array magnitude) for the 4-chimpanzee group, $-.13, R^{2}=$ $.16, F(1,8)=2.76, n s$, and an equal but opposite slope for Bobby, $-.13, R^{2}=.06, F(1,8)=0.96$, ns. These functions accounted for only $16 \%$ and $6 \%$ of variance, respectively, and in neither case were significant. Thus, the element size of arrays appeared to bias responding, regardless of whether the array with the larger elements had the larger or smaller magnitude. This was clearly illustrated on trials where one array contained a single large candy and the other was composed of a variable number of small 
candies (one to five). Again, regression analyses failed to reveal significant relations between performance and disparity ratio for either Bobby or the remaining chimpanzees $\left(R^{2} s<.05, n s\right)$. Instead, the predominant selection by the 4-chimpanzee group was the single large candy array, regardless of array magnitude. Bobby showed the opposite bias, selecting the array with smaller candy elements, again regardless of array magnitudes. Table 1 illustrates results with combinations of one large and two to five small candies, which are especially relevant because the single large candy had a greater mass than two or three small candies but a lesser mass than four or five candies. Bobby showed an opposite bias away from the array with the large element(s), instead selecting it on the corresponding trials an average of only $24 \%$ and $40 \%$, respectively. Although there did appear to be a slightly reduced bias for Bobby when the array with the larger elements also had the smaller magnitude, in neither case did this effect achieve significance. Element size was also a more potent determinant than quantity, as the array with the larger elements was selected regardless of whether it contained more or fewer candies. For the 4-chimpanzee group, the large element array was selected on $75 \%$ of trials where it contained the larger number and $85 \%$ of trials where it had the smaller quantity, $x^{2}(1, N=271)=3.7, n s$. Bobby once more showed the opposite pattern, selecting the large element array on $8 \%$ and $21 \%$ of the corresponding trials, respectively, $X^{2}(1, N=68)=3.2, n s$.

Despite these differences in the direction of bias, these results illustrate a potent response bias introduced by element size that was apparent in the performance of all chimpanzees tested. Four chimpanzees showed a distinct bias toward selection of arrays comprising large elements, regardless of array magnitude, even though it led to reduced rewards. Element size was also the predominant determinant in the single male's responses (Bobby), but in his case, the bias was away from selection of the large element array.

Table 1. Probability of Selecting a Single Element Array

\begin{tabular}{|lcc|}
\hline Trial type & Group $(\boldsymbol{n}=4)$ & Bobby \\
\hline $1 \mathrm{~L} / 2 \mathrm{~S}+1 \mathrm{~L} / 3 \mathrm{~S}$ & $.79^{\star^{\dagger}}$ & $.25^{\star}$ \\
$1 \mathrm{~L} / 4 \mathrm{~S}+1 \mathrm{~L} / 5 \mathrm{~S}$ & $.56^{\dagger}$ & .25 \\
Total $1 \mathrm{~L} / n \mathrm{n}$ trials & $.70^{\star}$ & $.25^{\star}$ \\
\hline
\end{tabular}

Note. $\mathrm{L}=$ large candy; $\mathrm{S}=$ small candy

* Significantly different from chance (i.e. 50), $p<.05$

${ }^{\dagger}$ Significantly different from each other, $p<.05$.

\section{$\underline{\text { Discussion }}$}

The results of the present study replicate and extend previous findings from our laboratory on a similar interference task (Boysen \& Berntson, 1995; Boysen et al., 1996) and illuminate the manner by which chimpanzees integrate size and number in quantity judgments. Our previous findings indicated that chimpanzees are highly sensitive to element quantity in quantity judgments of object arrays, consistent with the previous observations of Menzel and colleagues (e.g., Menzel, 1960; Menzel \& Draper, 1965). These studies also revealed that chimpanzees were unable to respond optimally under conditions of reversed reward contingencies when required to select the smaller object array to receive the larger reward. The predisposition to select the larger choice stimulus, however, could be overridden if symbolic representations for array quantities, in the form of Arabic numerals, were substituted for the object arrays. Together, these results suggest that some aspect of the perceptual or incentive features of the object arrays introduced an interfering bias in array choices. 
In the present study, we further examined the relative contributions of array quantity, element size, and total array mass on the interference effect. These findings document that chimpanzees were sensitive to array element size and total array mass in their choices. However, element size exerted a disproportionate influence relative to element quantity and total array mass. As we have previously observed, most chimpanzees displayed suboptimal performance under the reversed reward contingency, generally selecting the array with a larger mass and thus receiving the smaller reward (the unselected array). Moreover, chimpanzees tended to select arrays comprising larger candy elements, regardless of whether that array had a greater or lesser total mass. The presence of even one larger candy item alone as a choice produced a significant interference effect such that 4 of the 5 chimpanzees tested chose the array with the larger element(s), despite subsequently receiving a diminished reward.

Although array quantity and element size had a comparable impact on response choices for all chimpanzees, 1 chimpanzee displayed an opposite pattern of selection. Four of the 5 chimpanzees observed evidenced suboptimal performance, consistent with what we have observed in previous studies. That is, they generally selected the larger array, despite the fact that this response yielded the smaller reward. This bias was observed throughout testing with these chimpanzees, although 1 chimpanzee, as we have noted, was able to optimize reward by selecting the array with the smaller physical mass and garnered the larger, remaining array as a reward. The choices of this chimpanzee on selected trials were informative. Among the other 4 chimpanzees, element size predominated as a determinant of array choice. In contrast, the more successful chimpanzee responded suboptimally only on trials in which the array with the larger elements contained the smaller mass. That is, this chimpanzee tended to avoid selecting the array with the larger element, consistent with a disproportionate contribution from element size.

At the present time, it is unclear what mitigating factors may have permitted this single chimpanzee to achieve success on this version of the quantity judgment task. The remaining 4 chimpanzees, as well as the currently successful chimpanzee, have an extensive history of suboptimal responding on versions of the task that were based on quantity differences between the comparison arrays, and all chimpanzees had undergone repeated testing on numerous versions of the task over the past several years. Overall, all 5 chimpanzees tested in the previous experiments demonstrated consistently poor performance at remarkably comparable levels (see, e.g., Boysen et al., 1996). Further study may help to clarify performance differences in the present version of the task and may provide additional insights into the unique behavioral biases and predispositions revealed by quantity judgments in chimpanzees.

In summary, perceptual contributions to quantity judgments under the present reinforcement conditions appear to have a powerful impact on array choice by the chimpanzees. In addition to response biases arising from quantity differences between the comparison arrays, which replicate and extend previous findings, the present results document the impact of element size within an array. However, the chimpanzees did not appear to appropriately weigh or integrate size and quantity to derive an accurate estimate of total array mass. Rather, element size alone appeared to predominate as a determinant of response choice.

\section{Notes}

${ }^{1}$ Results were stable across sessions. Over all chimpanzees, the $r$ bias toward selection of the array with larger candy elements for choice pairs of equal quantity (e.g., two small and two large) was $63 \%$ for the first six sessions and $56 \%$ for the last five sessions, $X^{2}(1, N=100)=2.1, n s$. Similar results were obtained for matched discordant trial sets, where array quantity and element size would dictate opposite responses 
(e.g., for the choice pair of two small and one large, the array with one large candy had the smaller quantity but the larger element size). For these trials, chimpanzees selected the array with the larger elements on $68 \%$ of trials in the first six sessions and $66 \%$ of trials in the last five sessions, $X^{2}(1, N=339)$ $<1.0, n s$.

\section{References}

Anderson, J. R., Awazu, S., \& Fujita, K. (2000). Can squirrel monkeys (Saimiri sciureus) learn selfcontrol? A study using food array selection tests and reverse-reward contingency. Journal of Experimental Psychology: Animal Behavior Processes, 26, 87-97.

Boysen, S. T. (1997). Representation of quantities by apes. Advances in the Study of Behavior, 26, 435462.

Boysen, S. T., \& Berntson, G. G. (1995). Responses to quantity: Perceptual versus cognitive mechanisms in chimpanzees (Pan troglodytes). Journal of Experimental Psychology: Animal Behovior Processes, 21, 82-86.

Boysen, S. T., Berntson, G. G., Hannan, M. B., \& Cacioppo, J. T. (1996). Quantity-based interference and symbolic representations in chimpanzees (Pan troglodytes). Journal of Experimental Psychology: Animal Behavior Processes, 22, 76-86.

Boysen, S. T., Berntson, G. G., \& Mukobi, K. L. (1999). Overcoming response bias using symbolic representations of number by chimpanzees (Pan troglodytes). Animal Learning \& Behavior, 27, 229-235.

Ernrnerton, J. (1995, November). Biases in pigeons' responses to quantity: Discrimination of "more" vs. "less." Paper presented at the annual meeting of the Psychonornic Society, Los Angeles, CA.

Kreps, D. M. (1988). Notes on the theory of choice. Boulder, CO: Westview. Kuhlrneier, V. A., Boysen, S. T., \& Mukobi, K. L. (1999). Comprehension of scale models by chimpanzees (Pan troglodytes). Journal of Comparative Psychology, 113, 396-402.

Menzel, E. W., Jr. (1960). Selection of food by size in the chimpanzee and comparison with human judgments. Science, 131, 1527-1528.

Menzel, E. W., Jr. (1961). Perception of food size in the chimpanzee. Journal of Comparative and Physiological Psychology, 54, 588-591.

Menzel, E. W., Jr., \& Davenport, R. K., Jr. (1962). The effects of stimulus presentation variables upon chimpanzee's selection of food by size. Journal of Comparative and Physiological Psychology, $55,235-239$.

Menzel, E. W., Jr., \& Draper, W. A. (1965). Primate selection of food by size: Visible versus invisible rewards. Journal of Comparative and Physiological Psychology, 59, 231-239.

Mischel, W., Shoda, Y., \& Rodriguez, M. L. (1989, May 26). Delay of gratification in children. Science, 244, 933-938.

Schrier, A. M. (1956). Amount of incentive and performance on a black-white discrimination problem. Journal of Comparative and Physiological Psychology, 43, 123-125.

Silberburg, A., \& Fujita, K. (1996). Pointing at smaller food amounts in an analogue of Boysen and Berntson's (1995) procedures. Journal of the Experimental Analysis of Behavior, 66, 143-147. 